\title{
Self-organization of polyaniline nanorods: Towards achieving a higher conductivity
}

\author{
Debangshu Chaudhuri, Suwarna Datar, Ranjani Viswanatha, and D. D. Sarma ${ }^{\text {a) }}$ \\ Solid State and Structural Chemistry Unit, Indian Institute of Science, Bangalore-560012, India \\ Heinz Amenitsch \\ Institute of Biophysics and X-ray Structure Research, Austrian Academy of Sciences, Schmiedlstr. 6, \\ 8043 Graz, Austria
}

\begin{abstract}
We present a scanning tunnelling microscopy and spectroscopy study of polyaniline nanostructure. Our results show the possibility of achieving a high degree of self-organization in polyaniline nanorods in the undoped as well as the doped states. The spectroscopy results clearly establish a direct correlation between the extent of ordering and the density of states at the Fermi energy of the doped samples. Small angle x-ray scattering experiment helps to understand the formation of such self-organized structures.
\end{abstract}

Research in the field of molecular electronics has gained significant momentum in the recent past and conducting polymers are expected to play an important role in the further development of this field. Among the various members of this family, polyaniline (PANI) has been one of the most widely studied conducting polymers. Over the last decade, there have been numerous efforts in developing newer systems with improved properties, based on PANI. ${ }^{1-3}$ However, the key to exploit the true potential of these materials lies in achieving highly organized structures at nanometer length scales. Recently, there have been several reports on the synthesis of microns long PANI nanofibers with very uniform diameters in the range of $30-340 \mathrm{~nm}^{4,5}$ Although, the higher surface area of these nanofibers ensures faster response in sensory applications, their conductivity remains less than that of PANI prepared through conventional routes. This may be due to a poor morphological order of such nearly macroscopic polymer rods. Recent reports of scanning tunneling microscopy (STM) and atomic force microscopy (AFM) studies on various proton-doped PANI reveal the granular morphology of these systems. ${ }^{6-9}$ In certain cases, a few isolated PANI nanoropes of $\sim 50 \mathrm{~nm}$ diam have also been observed. ${ }^{9}$ However, current images show that these nanoropes are not homogenously doped, thereby suggesting a poor sample quality. Therefore, to make PANI a viable choice from the point of view of fabricating nanoscale devices, it is essential to have polymer chains effectively doped and simultaneously ordered at nanometer length scales. The effect of self-organization of PANI nanorods on its conductivity has been demonstrated in the complexes of camphorsulphonic acid (CSA) doped PANI with 4-hexyl resorcinol. ${ }^{10}$

In this article, we show with the help of STM studies that solution processing of undoped PANI can help to achieve a very high degree of self-organization at nanometer length scales. Further, we report on the effect of various dopants and doping procedures on the morphology of polymer thin films. Scanning tunneling spectroscopic (STS) measurements establish a higher density of states in the mid-gap region for the more ordered samples, thereby providing a

\footnotetext{
${ }^{a}$ Also at: Jawaharlal Nehru Centre for Advanced Scientific Research, Ban-
} galore, India; electronic mail: sarma@sscu.iisc.ernet.in direct explanation for their higher conductivities. With small angle x-ray scattering (SAXS) studies, we establish the preexistence of multichain nanorods in the solution. Further alignment of these highly anisotropic nanorods is presumably achieved during the course of making the thin-film sample.

STM imaging and spectroscopy were performed on Omicron VT AFM 25 DRH in air and at the room temperature $(\sim 300 \mathrm{~K})$. Mechanically cut Pt-Ir tips were used for the experiments. For the microscopic studies, thin films were deposited by spin coating on freshly cleaved highly oriented pyrolytic graphite (HOPG) substrate. Polyaniline was synthesized by oxidative polymerization of aniline using $\left(\mathrm{NH}_{4}\right)_{2} \mathrm{~S}_{2} \mathrm{O}_{8}$ at $0-5{ }^{\circ} \mathrm{C} .{ }^{11}$ A $3 \%$ w/v solution of undoped PANI in N-methyl pyrrolidone (NMP) was used. $\mathrm{HCl}$ doping was carried out by immersing the undoped film in $1 \mathrm{M} \mathrm{HCl}$ solution for ten hours. Doping with CSA was carried out in two different fashions. CSA-1 was prepared as a thin film from a $3 \% \mathrm{w} / \mathrm{v}$ solution of $50 \%$ doped CSA-PANI in $m$-cresol. CSA-2 was prepared in a way similar to $\mathrm{HCl}$ doped PANI, by immersing an undoped thin film in $1 \mathrm{M}$ aqueous solution of CSA. For the spectroscopic studies, relatively thicker films were made by drop casting. SAXS experiments were performed at the Austrian SAXS beamline installed at the $2.4 \mathrm{GeV}$ electron storage ring, ELETTRA, Trieste, Italy. ${ }^{12}$

STM image of a spin-coated undoped PANI film shows a highly organized morphology [see Fig. 1(a)]. We find cylindrical rods with diameters ranging between 5 and $10 \mathrm{~nm}$ aligned parallel to each other. In certain regions of the sample, we have observed such an alignment over a length of about $600 \mathrm{~nm}$. Since the width of an isolated polymer molecule is of the order of $6 \AA$, the image suggests that each rod represents a bunch of several polymer chains aligned longitudinally. Further, we note that the thin films show no trace of granular structures. It is well-known that the strong intraand inter-molecular H-bonding interactions in PANI can lead to extensive coiling of the polymer chains resulting in granular morphology. In such a coiled configuration, the planarity of the backbone is lost, which is expected to have an adverse effect on the $\pi$-electron delocalization. In dilute solutions of PANI in NMP, the strong polymer-solvent H-bonding helps 


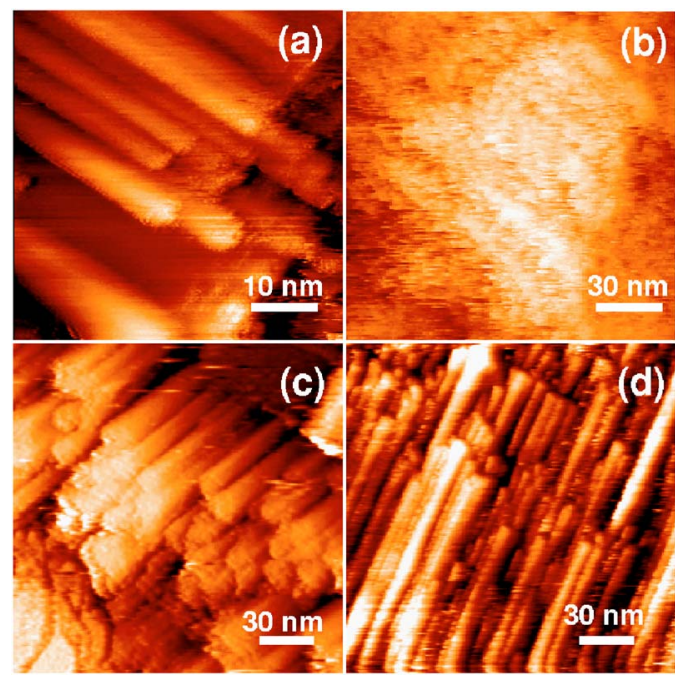

FIG. 1. (Color online) The STM images of (a) undoped PANI, (b) HCldoped, (c) CSA-1, and (d) CSA-2 PANI.

to open up these coiled-up chains and achieve a "chainextended" configuration, as can be seen from the figure. While in such a configuration, the electron delocalization along a single chain is much more facile, it also helps the individual chains to interact better among themselves, leading to a more efficient three-dimensional delocalization of electronic wave functions. Thus, one might expect to achieve very high conductivities provided such a high degree of ordering is retained after doping.

STM images in Fig. 1(b)-1(d) show the effect of various dopants and doping procedures on the structural ordering in PANI. Upon HCl-doping, we observe that the ordering is almost entirely lost [see Fig. 1(b)]. Perhaps one can still see the remnant of an ordered structure. However, the rod-like moieties can no longer be seen and the sample appears predominantly granular, in agreement with previous results. ${ }^{7}$ The fact that starting with an ordered, well-aligned undoped PANI, one ends up having a disordered structure in the doped state suggests that the dopant has a very important role to play in determining the final morphology of the sample. This is further corroborated by our findings with the CSA-1 sample. Once again the effect of solution processing is quite evident, as we observe bundles of cylindrical nanorods aligned parallel to each other [see Fig. 1(c)]. The presence of such ordered morphology in solution-processed CSA-doped PANI in contrast to the disordered granular structure of $\mathrm{HCl}-$ PANI also explains the difference in the corresponding conductivities and its temperature dependence. ${ }^{13}$ We now turn our attention to the STM image of CSA-2 sample [Fig. 1(d)]. In spite of the fact that this sample was prepared in a manner similar to the HCl-PANI, by post-doping an undoped thin film we find a significant degree of ordering over length scales as large as $200 \mathrm{~nm}$. This observation establishes that an appropriate choice of dopant helps to retain the ordering present in the undoped PANI.

We have measured the $I-V$ characteristics of the samples using the STM tip. The $I-V$ curves obtained from different spots on a particular sample were identical, indicating sample homogeneity. It is well-known that one can obtain valuable information about the local density of states (DOS) from $d I / d V$ curves. Further, $d I / d V$ at $V=0$ provides a measure of the DOS at the Fermi level $\left(E_{F}\right) \cdot{ }^{14}$ In Fig. 2, we

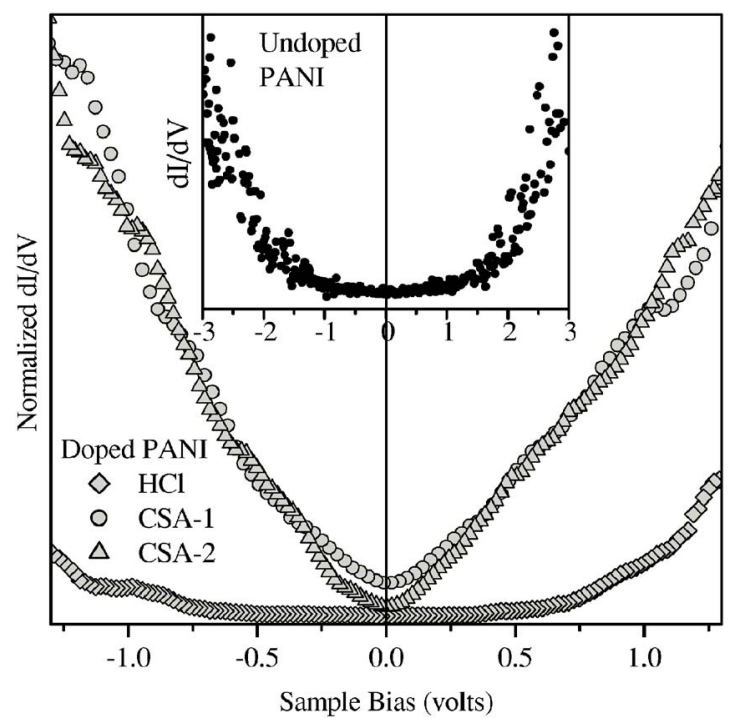

FIG. 2. Plot of $(d I / d V)$ as a function of the sample bias, $V$, for the three doped PANI samples; $\mathrm{HCl}(\diamond), \mathrm{CSA}^{-1}(\bigcirc)$, and $\mathrm{CSA}-2(\triangle)$. The inset shows the data for undoped PANI.

present the results of tunneling spectroscopy for different samples. The inset shows the $d I / d V$ for undoped PANI. One can see a band gap of about $3.5 \mathrm{eV}$, consistent with the value obtained from uv-visible absorption spectroscopy. ${ }^{1}$ Increases in the $d I / d V$ at extreme voltages correspond to the valence band (VB) and the conduction band (CB) edges. The main panel of Fig. 2 shows the normalized $d I / d V$ for $\mathrm{HCl}$, CSA-1, and CSA-2 doped samples. Considering the fact that doping primarily introduces states in the gap, we have normalized the individual $d I / d V$ curves for the doped samples near the CB edge $(+2.1 \mathrm{~V}) .{ }^{15}$ Compared to undoped PANI, the $\mathrm{HCl}-$ PANI has a substantially higher DOS in the gap. However, one can still see a gap-like structure of $\sim 1 \mathrm{eV}$ width near $E_{F}$. CSA-1 and CSA-2 samples, on the other hand, show very different $d I / d V$. It is evident that the gap-like structure near $E_{F}$ vanishes completely in these two samples. Further, in contrast to the $\mathrm{HCl}$-doped PANI, $d I / d V$ at $V=0$ for CSA-1 and CSA-2 samples are about 2.5 and 6 times higher, respectively. These results suggest that an ordered structure of CSA-doped PANI ensures a higher DOS in the mid-gap region, and a finite DOS at the $E_{F}$. On the contrary, $\mathrm{HCl}$ doping destroys the nanoscopic ordering and, consequently, have a much lower DOS in the mid-gap region and at the $E_{F}$. Despite an identical doping procedure for $\mathrm{HCl}$ and CSA-2 PANI, retention of the ordered structure in thin-film of the latter correlates well with its gapless electronic structure. Among the two CSA-doped samples, a higher DOS at the $E_{F}$ for CSA-1 sample is presumably because of the post-doping solution processing that helps to achieve an extensive ordering throughout the bulk. Since no such post-doping solution processing is done for the CSA-2 sample, it possibly suffers from a partial loss of ordering that was originally present in the undoped PANI film. A lower DOS at the $E_{F}$ for CSA-2 sample can also arise from an incomplete doping in the core region of these nanorods.

In order to understand the mechanism by which these ordered nanostructures form in the solution-processed films, we carried out SAXS measurements on 3\% solution of CSAdoped PANI in $m$-cresol. Figure 3 shows a typical SAXS pattern. In the Guinier regime $\left(q * R_{G} \ll 1\right)$, the scattered in- 


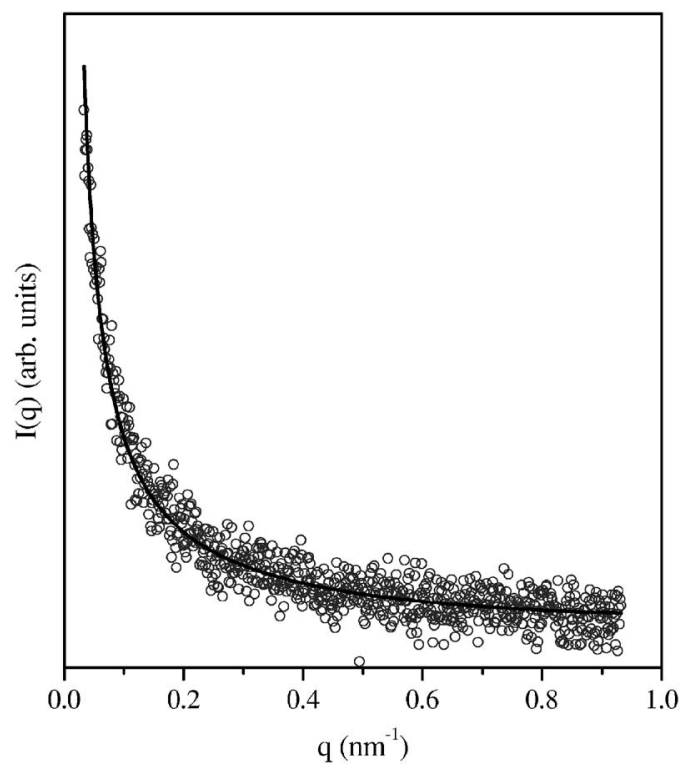

FIG. 3. Variation of SAXS intensity as a function of the scattering vector. The solid line shows the least-squared fit assuming rod-like particles, using Guinier's law.

tensity for infinitely long rod-like particles is given by ${ }^{16}$ $I(q) \propto 1 / q \times \exp \left(-q^{2} R_{G}^{2} / 2\right)$, where $R_{G}$ is the cross-sectional Guinier's radius. The assumption of infinitely long rods is valid in our experiment as the length of the nanorods are much larger than the instrumental resolution of about $25 \mathrm{~nm}$. For infinitely long monodisperse rods of radius $R$, the Guinier's radius $\left(R_{G}\right)$ is given by $R / \sqrt{2}$. In Fig. 3 we show the least-squared fit for the SAXS intensity using the above expression for $I(q)$. The diameter $(2 R)$ of the rods evaluated from the $R_{G}$ value is $7.7 \mathrm{~nm}$, which agrees remarkably well with the diameter of $10 \mathrm{~nm}$ observed from STM [see Fig. 1(c)]. This agreement with the Guinier's law ( $q$ $<0.36 \mathrm{~nm}^{-1}$ ) establishes the existence of PANI nanorods in the solution. During the preparation of thin film, as the solvent evaporates and the concentration increases, these nanorods come closer and begin to self-assemble. The difference in the morphology of the $\mathrm{HCl}$-doped and CSA-2 thin film samples can be explained in terms of the interactions between the respective counterions and the doped polymer. In the absence of any solvent, the final morphology of the doped film is expected to be governed by the competition between the polymer-polymer and polymer-counterion interactions. Since the carbonyl group $(\mathrm{C}=\mathrm{O})$ of the camphorsulfonate counterion can form $\mathrm{H}$-bond with the doped polymer molecule, it presumably stabilizes the original or- dered structure of the undoped film, even after doping. In absence of such interactions, $\mathrm{HCl}$ doped PANI undergoes significant structural reorganization that is primarily driven by the intra- and inter-chain interactions.

To conclude, we have prepared undoped PANI films with highly aligned nanorods over large length-scales. With a suitable choice of dopants and the doping procedure, we have achieved homogenous doping and a high degree of ordering in the doped samples. STM and STS studies establish that such highly organized morphology is essential to having a finite DOS at $E_{F}$. SAXS results establish that the polymer nanorods are preformed in solution. The highly anisotropic aspect ratios of these nanorods leads to their selforganization upon thin film formation.

This work was supported by the Department of Science and Technology (DST) and the International Centre for Theoretical Physics under the ICTP-Elettra users program for synchrotron radiation. The authors are thankful to Angshuman Nag for help in synchrotron experiments.

${ }^{1}$ D. Chaudhuri, A. Kumar, I. Rudra, and D. D. Sarma, Adv. Mater. (Weinheim, Ger.) 13, 1548 (2001).

${ }^{2}$ D. Chaudhuri, A. Kumar, D. D. Sarma, M. García-Hernández, J. P. Joshi, and S. V. Bhat, Appl. Phys. Lett. 82, 1733 (2003).

${ }^{3}$ D. Chaudhuri, P. W. Menezes, and D. D. Sarma, Appl. Phys. Lett. 83, 2348 (2003).

${ }^{4}$ J. Huang and R. B. Kaner, J. Am. Chem. Soc. 126, 851 (2004).

${ }^{5}$ Z. Zhang, Z. Wei, and M. Wan, Macromolecules 35, 5937 (2002).

${ }^{6}$ P. K. Ho, P. C. Jhang, L. Zhou, S. F. Y. Li, and H. S. O. Chen, Phys. Rev. B 56, 15919 (1997).

${ }^{7}$ S. T. Yau, J. N. Barisci, and G. M. Spinks, Appl. Phys. Lett. 74, 667 (1999).

${ }^{8}$ M. Wan, C. Zhu, J. Yang, and C. Bai, Synth. Met. 69, 157 (1995).

${ }^{9}$ D-.H. Han and S-.M. Park, J. Phys. Chem. B 108, 13921 (2004).

${ }^{10}$ H. Kosonen, J. Ruokolainen, M. Knaapila, M. Torkkeli, K. Jokela, R. Serimaa, G. ten Brinke, W. Bras, A. P. Monkman, and O. Ikkala, Macromolecules 33, 8671 (2000).

${ }^{11}$ A. G. MacDiarmid, J. C. Chiang, A. F. Richter, and A. J. Epstein, Synth. Met. 18, 285 (1987).

${ }^{12}$ H. Amenitsch, S. Bernstorff, M. Kriechbaum, D. Lombardo, H. Mio, M. Rappolt, and P. Laggner, J. Appl. Crystallogr. 30, 872 (1997).

${ }^{13}$ R. Menon, Y. Cao, D. Moses, and A. J. Heeger, Phys. Rev. B 47, 1758 (1993).

${ }^{14}$ R. J. Hamers, in Scanning Tunneling Microscopy and Spectroscopy: Theory, Techniques, and Applications, edited by D. A. Bonnell (VCH, Weinheim, 1993), p. 51.

${ }^{15} \mathrm{On}$ the basis of semi-empirical band structure calculations, it has been argued that doping in PANI introduces new states at the top of the VB. This was primarily the reason for our carrying out the normalization at the $\mathrm{CB}$ edge. However, on carrying out the normalization well inside the VB, the conclusions remain essentially the same.

${ }^{16} \mathrm{G}$. Porod, General theory. In small angle x-ray scattering (Academic, London, 1982). 